\title{
Pulsed laser deposition of indium antimonide ${ }^{\dagger}$
}

\author{
R VENKATARAGHAVAN, K M SATYALAKSHMI*, K S R K RAO, \\ A K SREEDHAR, M S HEGDE* and H L BHAT \\ Department of Physics, *Solid State and Structural Chemistry Unit, Indian Institute of \\ science, Bangalore 560012 , India
}

\begin{abstract}
1bstract. Single crystalline oriented films of indium antimonide have been grown on cadmium telluride substrates by the pulsed laser deposition technique. The films were (111) uriented which is the substrate orientation. The composition of the grown films were found to deviate from that of the target owing to loss of antimony during evaporation. This deviation irom stoichiometry led to film-substrate reaction, resulting in mixed interface. The antimony Jeficiency in the films were controlled by correcting the stoichiometry, which led to avoiding mixed interfaces. The stoichiometric films showed good surface morphology and well defined sharp interfaces. The IR transmission spectrum showed sharp band to band absorption and iffective detection in the MWIR.
\end{abstract}

Keywards. PLD: indium antimonide; morphology; interface; $\mathrm{In}_{2} \mathrm{Te}_{3} ; \operatorname{InTe}$.

\section{Introduction}

Indium antimonide, a narrow band gap III-V compound semiconductor, finds application as IR detectors. Its band gap absorption corresponds to the atmospheric transparency window between 3-5 $\mu \mathrm{m}$ (MWIR). Numerous advances have been made in the commercial fabrication of InSb detectors. Epitaxial layers on suitable substrates have been grown, including $\mathrm{InSb} / \mathrm{CdTe}$, which are a new generation of III-V/II-VI structures that form type I heterojunctions. Their perfect lattice match, large band offsets and compatible thermal properties have promoted extensive research investigations on their growth and properties. Reports of growth by vacuum evaporation (LeFloch 1968; Rabin et al 1980) and films grown by the temperature gradient vapour transport deposition (TGVTD) by Kim et al (1992) have been discouraging owing to the mixed interfaces due to the high annealing temperatures employed in these processes. In spite of its perfect lattice match to CdTe, the growth of InSb films has not been easy due to the formation of incoherent interfaces, that has hindered device fabrication. Even in the growth of InSb/CdTe by MBE (Sugiyama 1982; Williams et al" 1985), it was difficult to avoid diffused interfaces. The invariable occurrence of a mixed layer at the film-substrate junction has drawn considerable attention to the study of the interfaces. The compound formed at the interface has been identified as indium telluride $\left(\mathrm{In}_{2} \mathrm{Te}_{3}\right)$ by Zahn et al (1989) who used Raman spectroscopy and independently by Kim et al (1992). However, a report by van Welzenis et al (1991), attributes it to a stressed InTe phase. They suggest a low temperature, high growth rate process to alleviate the interface problem during growth. A process like the pulsed laser deposition (PLD) satisfies the conditions of low temperature and high growth rate. Except for an early attempt to grow InSb by PLD (Gaponov et al 1980), to date there has not been any other report of laser deposited films of this material. We report here the growth of

\footnotetext{
'Paper presented at the poster session of MRSI AGM VI, Kharagpur, 1995
} 
oriented films of indium antimonide on cadmium telluride substrates by the pulsed laser deposition technique.

\section{Experimental}

Thin layers of InSb were grown on (111) semi-insulating CdTe substrates by the pulsed laser deposition technique. The CdTe substrates used in the present investigation were supplied by Solid State Physics Laboratory, Delhi. Samples were cut to the size of $10 \times 5 \mathrm{~mm}^{2}$ from $1.5 \mathrm{~mm}$ thick (111) wafers and polished to a surface finish of $0.1 \mu \mathrm{m}$. The films were deposited on the CdTe (111) A surface. The material to be evaporated, $\mathrm{InSb}$, was taken in the form of a disc, $19 \mathrm{~mm}$ across and $7 \mathrm{~mm}$ thick. The compound was synthesized using $5 \mathrm{~N}$ purity indium and antimony, procured from the Nuclear Fuel Complex Ltd., Hyderabad, by alloying them at a temperature of $800^{\circ} \mathrm{C}$ in an axially rotating homogenizing furnace, and quench cast into a disc. The substrate surfaces were cleaned sequentially by tri-chloro ethylene (TCE), acetone and methanol. The InSb target was also subjected to the same cleaning procedures and further followed by cleaning in bromine-methanol and then rinsing in methanol.

A Lambda Physik KrF pulsed excimer laser, lasing at $248 \mathrm{~nm}$ was used for evaporation. Laser energy of $280 \mathrm{~mJ} /$ pulse with an energy density of $1.5 \mathrm{~J} / \mathrm{cm}^{2}$ was made to impinge on the target at a repetition rate of $5 \mathrm{~Hz}$ for a total of 5000 pulses. The substrate was positioned at the centre of the resulting plume to avoid lateral inhomogeneity. The evaporation process was carried out at a substrate temperature of $225^{\circ} \mathrm{C}$ in $10^{-5}$ torr vacuum for a total of $16 \mathrm{~min}$. In order to assess the compositional homogeneity and to determine the composition of the deposited film, an EDAX of the film was recorded using a SPEC system from Cambridge Instruments. A depth concentration profile was recorded to determine the distribution of the various atomic species across the film substrate interface using a CAMECA IMS4F SIMS profiler. A Leitz polarizing optical microscope was used to study the surface morphology and the film-substrate interface. To study the interface, the samples were edge polished to $1 \mu \mathrm{m}$ finesse. The film-substrate interface did not require differential staining as it was easily distinguishable. Photographs were taken with the attached camera, in phase contrast mode. Occasionally, the interfaces were also photographed using a SEM. $X$-ray diffraction patterns were recorded using a computer controlled JEOL X-ray diffractometer. The IR transmission spectra for the grown structures were recorded at room temperature using a Shimadzu IR 435 spectrometer in the region of interest. The region scanned corresponds to the MWIR, where the absorption is due to the film but the incident radiation is completely transmitted through the substrate.

\section{Results and discussion}

The grown films were first subjected to microscopic observation. As can be seen from figure 1a, the film shows matty surface morphology. This is typical of laser assisted epitaxy and occurs because of the superheating of the subsurface before the surface has reached vapour phase. The ablated particles can then get trapped inside the subsurface layer leading to perturbed crystallinity during growth (Cheung and Magee 1983). This leads to low surface coverage of the substrate and porosity in the grown film. The surface morphology can however be improved by reducing the energy fluence of the 

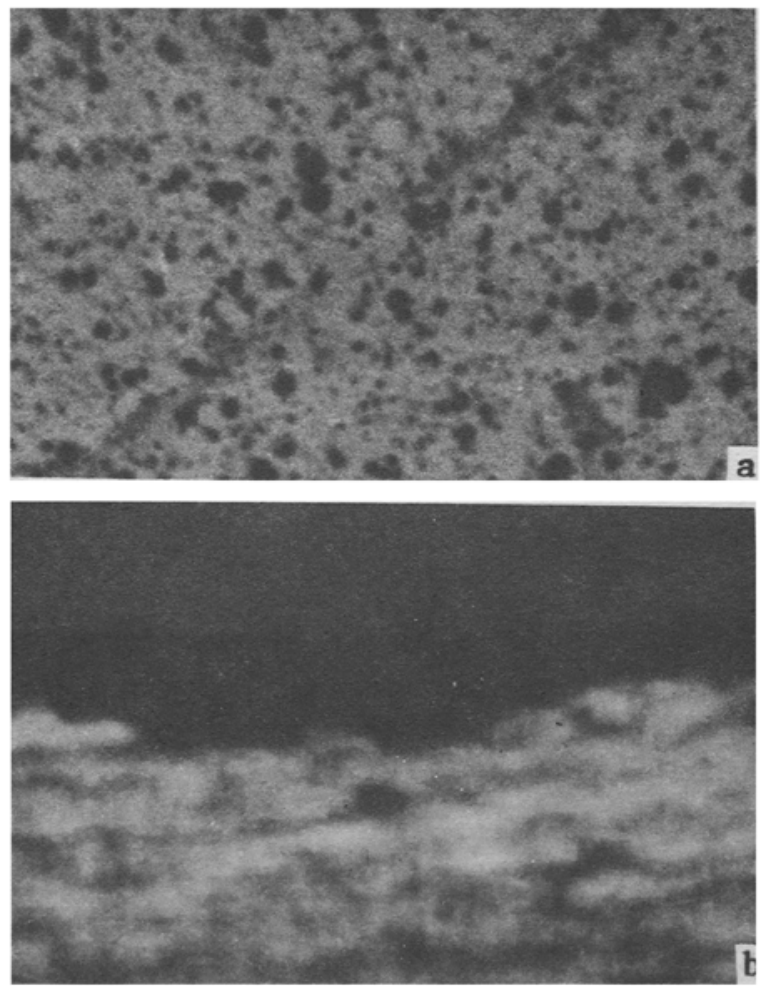

Figure 1. (a) Optical micrograph of InSb epilayer showing the surface morphology $(\times 667)$ and (b) edge-on view of the structure showing film-substrate interface $(\times 333)$.

lable 1. Results of EDAX on the target and the grown filn showing the , roichiometry.

\begin{tabular}{|c|c|c|c|}
\hline \multicolumn{2}{|c|}{ Target composition } & \multicolumn{2}{|c|}{ Film composition } \\
\hline Indium (at. \%) & Antimony (at.\%) & Indium (at.\%) & Antimony (at. \%) \\
\hline$+9 \cdot 62$ & $50 \cdot 38$ & $54 \cdot 16$ & $45 \cdot 84$ \\
\hline$+2 \cdot 62$ & $57 \cdot 38$ & $48 \cdot 34$ & 51.66 \\
\hline
\end{tabular}

laser source. Figure $1 \mathrm{~b}$ shows the edge-on view along the film. The micrograph clearly shows the poor quality of the interface. The film thickness is also seen to vary along the length of the interface by about $\pm 10 \%$. Some bright spots, not shown here, visible on the substrate side of the interface, were probably indium aggregates that had diffused into the substrate. The average film thickness based on microscopy was calculated to be around $14 \mu \mathrm{m}$.

A study of the results of EDAX (table 1), leads us to believe that the deposited film is indium rich. In order to confirm the stoichiometry in the target, it was subjected to EDAX, but stoichiometry was established from the analysis. This proved that there is a loss of antimony from the plume during the evaporation process. 


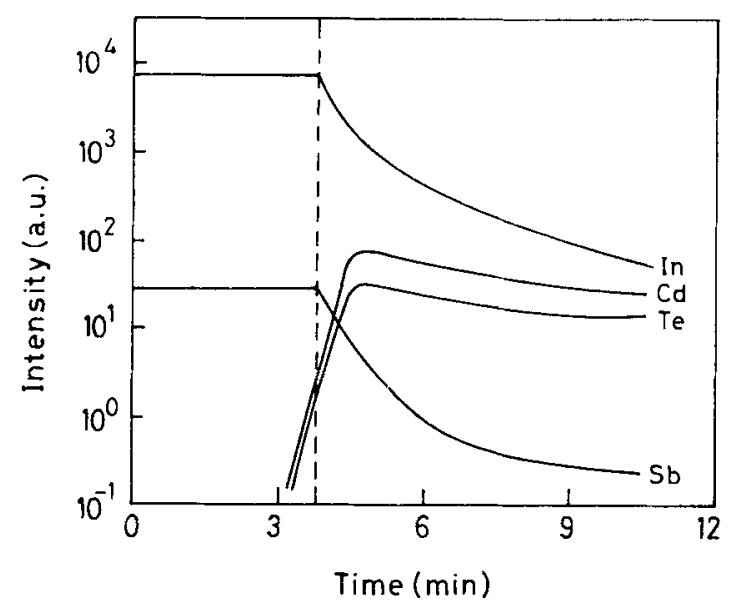

Figure 2. Atomic concentration depth profile of the structure across the interface.

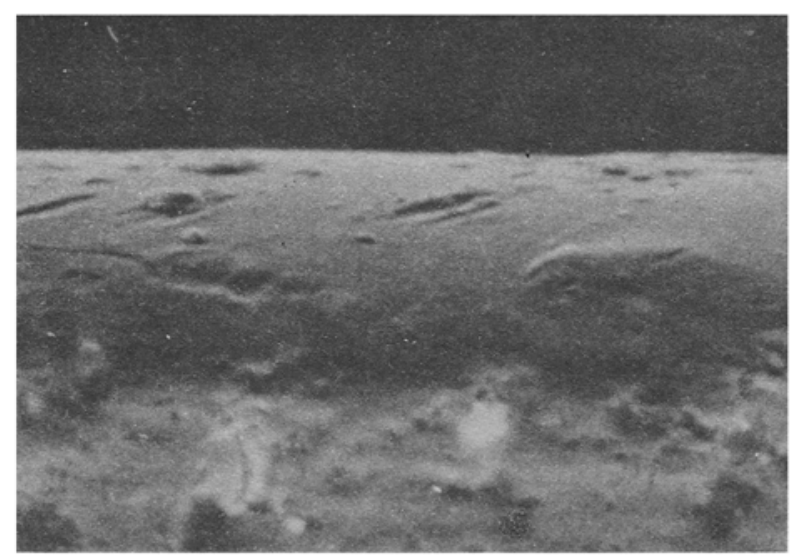

Figure 3. SEM showing interface for stoichiometric films $(\times 1000)$.

The SIMS profile in figure 2 shows the concentration of $\mathrm{In}, \mathrm{Sb}, \mathrm{Cd}$ and Te along the depth from the film. The drop-off of the indium signal into the substrate is a clear inverse exponential as given by the diffusion equation. This points to the diffusion of indium into the substrate and the presence of a thin layer along the interface where In, $\mathrm{Cd}$, and Te coexist. This was also encountered in films grown by Rabin et al (1980) by vacuum deposition.

Based on conclusions from analysis using EDAX, the antimony loss from the plume during evaporation was calculated. Using this, a target was cast as described earlier but with excess antimony so as to compensate for the antimony lost during the process of evaporation. Nearly stoichiometric films were grown using the antimony rich target (table 1). The layers grown with the antimony rich target, on observation using SEM (figure 3), showed sharp interfaces, in contrast to those grown from the stoichiometric target. Even under higher magnifications and better resolution achievable using SEM, interfacial feature like grooving of the film into the substrate and micro-aggregations were not revealed. The absence of the film grooving into the substrate at the interface, 


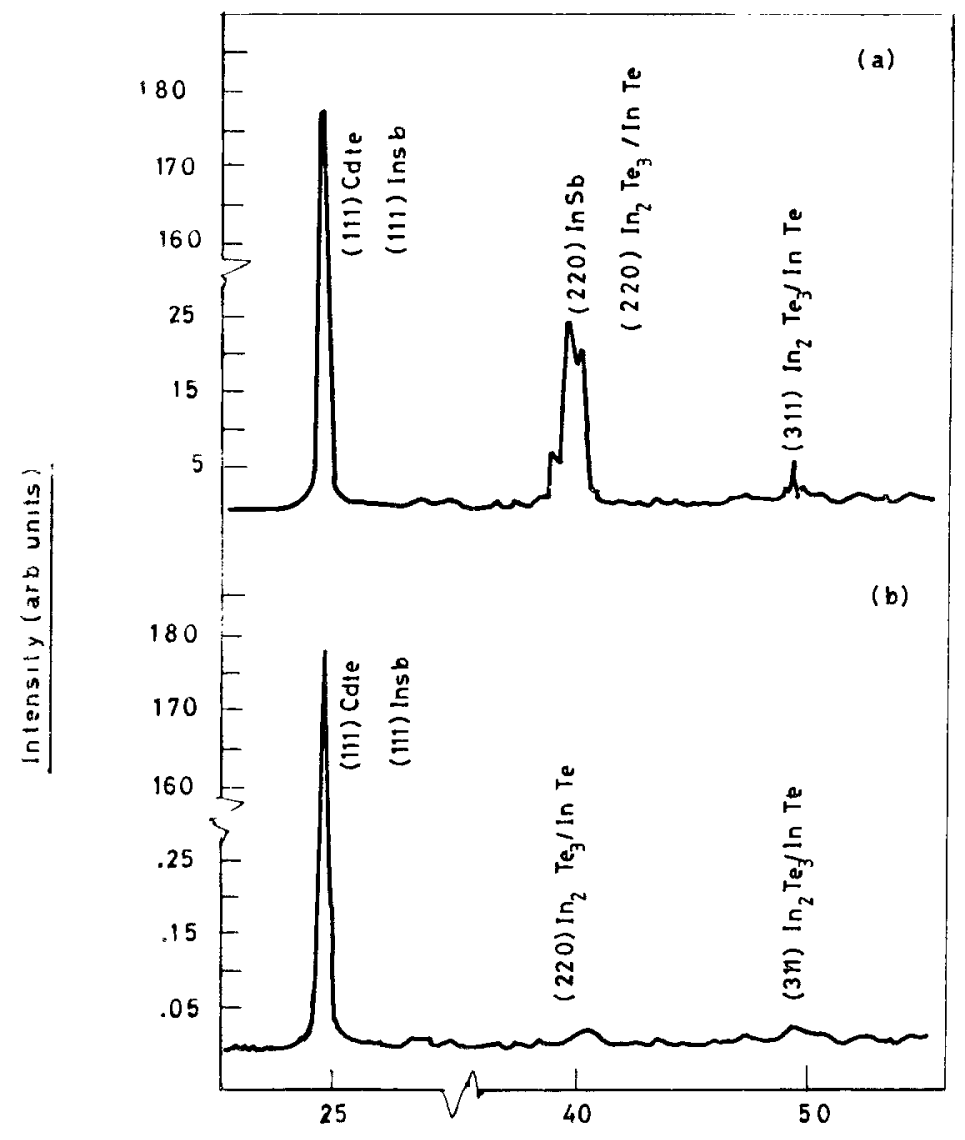

Figure 4. Diftracton patterns of (a) off-stoichiometric and (b) stoichiometric films.

shows the reduction in the interfacial reaction and the formation of interfacial aggregates. The existence of a clear demarcation of the film and substrate proves the superior quality of the interface. The thickness of the film using SEM was found to be about $16 \mu \mathrm{m}$. and was uniform along the length of the interface.

The X-ray diffraction pattern of the film grown from stoichiometric target (figure 4a). showed a dominant peak at $d$ value of $3.667 \AA$ which was attributed to the $\langle 111\rangle \mathrm{CdTe}$ peak because of its strength. A weaker peak at $2 \cdot 286 \AA$ was attributed to the $\langle 220\rangle$ plane of CdTe or InSb. It was not possible to distinguish between them due to their almost perfect lattice match (lattice mismatch $0.03 \%$ ). Two peaks at $d$ values $2.273 \AA$ and $1.858 \AA$ did not match either to InSb or CdTe. These could be indexed as $\langle 220\rangle$ and 311 $>$ planes of indium telluride $\left(\operatorname{In}_{2} \mathrm{Te}_{3}\right)$. However, van Welzenis et al (1991) attributed these to the stressed InTe phase. The diffraction pattern for the films grown from the antimony rich target (figure 4 b), showed only one dominant peak that indexed as the 111) plane of InSb. The absence of peaks attributed to the other phases of InSb or to $\mathrm{In}_{2} \mathrm{Te}_{3}$ confirms the single crystallinity in the grown film.

The room temperature IR transmission spectra, shown in figures $5 \mathrm{a}$ and $\mathrm{b}$ for samples grown from stoichiometric and antimony rich targets respectively, show the characteristic absorption band edge at $1650 \mathrm{~cm}^{-1}(\sim 6 \mu \mathrm{m})$ corresponding to the bandgap of InSb $(0.17 \mathrm{eV})$. In comparison to the spectrum of film grown from the 


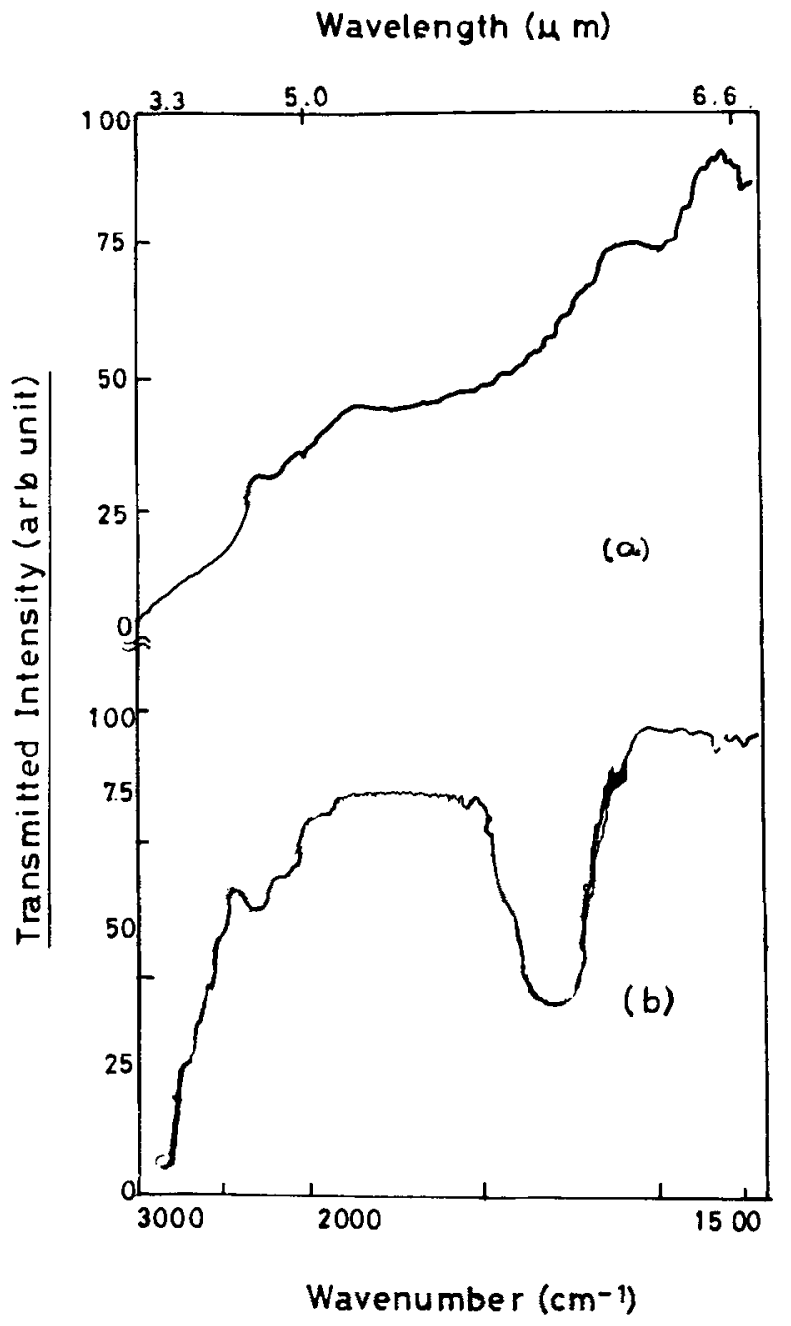

Figure 5. IR spectra in the MWIR of (a) off-stoichiometric and (b) stoichiometric films.

antimony rich target, the films grown from the stoichiometric target show a gradual fall and not a sharp onset of the band to band absorption. This may be due to scattering from a poorly formed interface in the former case. The transition in (b), grown from the antimony rich target, is however sharp, due to an aggregate free interface.

The results of EDAX and SIMS on the structure and the photoluminescence data (Suma et al 1994) on the substrate, support the availability of free indium and tellurium at the interface. The availability of free indium in the film, as is evident from EDAX, and existence of excess tellurium in the substrate, makes an interfacial reaction very favourable. This can be supported by thermo-energetic calculation of the probable reactions at the interface (Venkataraghavan et al 1996). The indium reacts with the free tellurium from the substrate to form an interfacial compound of indium telluride. This seems to be complementary to that reported in the growth of CdTe on InSb (Golding et al 1988), where a tellurium rich film is formed from stoichiometric molecular beams, which on reaction with indium forms $\mathrm{In}_{2} \mathrm{Te}_{3}$. 
The $\mathrm{X}$-ray diffraction pattern confirms the presence of an interfacial compound, and indexes as indium telluride $\left(\operatorname{In}_{2} \mathrm{Te}_{3}\right)$. Although the calculated lattice parameter matched to $\mathrm{In}_{2} \mathrm{Te}_{3}$, it may well compare to the lattice parameter of $\mathrm{InTe}$ in a stretched phase (van Welzenis et al 1991) as mentioned earlier. It is difficult to differentiate between these two phases of indium telluride based on diffractometry alone. Taking recourse to thermoenergetics and calculating the Gibbs free energy for the reactions involved at the interface, it is inferred that the $\operatorname{In}_{2} \mathrm{Te}_{3}$ phase is more likely to be present as against InTe (Venkataraghavan et al 1996). Hence, the reason for the formation of the interfacial compound is the free availability of indium at the interface which is due to the off-stoichiometry of the grown film.

Results of films grown from the antimony rich targets suggest that the film off-stoichiometry is the primary reason for the interfacial reaction. Films grown from the antimony rich target did not pose the problem of interface formation, exhibiting sharper interface. This was also shown by the diffraction patterns and the IR transmission spectra.

\section{Conclusion}

In conclusion, single crystalline $\langle 111\rangle$ oriented films of indium antimonide have been grown on cadmium telluride substrates by the pulsed laser deposition technique. By improving the growth conditions, it was possible to better the films by alleviating the interface problem by close monitoring and control of the film stoichiometry. Results of the diffractometry and IR spectroscopy prove the better interfacial quality of the films grown from off-stoichiometry targets. The long wavelength cut off of the heterostructure at $6 \mu \mathrm{m}$ suggests its utility as a detector in MWIR.

\section{Acknowledgement}

The authors acknowledge the help of Dr Vikram Kumar, Director, SSPL. Delhi, for access to the SIMS facility, Dr N V Madhusudana, Raman Research Institute, Bangalore for help in recording the IR transmission spectra and Mr Sam Philip for technical assistance with EDAX and SEM.

\section{References}

Cheung J T and Magee T 1983 J. Vac. Sct. Technol. Al 16014

Goponov S V, Luskin B M and Salaschenko N N 1980 Sov. Phys. Semicond. 14873

Golding T D, Martinka M and Dinan J H 1988 J. Appl. Phys. 641873

Kim T W, Jung M, Park H L, Na H K and Kim J S 1992 Appl. Phys. Lett. 611101

LeFloch G 1968 Thin Solid Films 2383

Rabin B, Scharager C, Hage-Ali M, Stiffert P, Wald F V and Bell R O 1980 Phys. Status Solidi A62 237

Sugiyama K 1982 J. Cryst. Growth 60450

Suma Gurumurthy, Rao K S R K, Sreedhar A K, Bhat H L, Sundersheshu B, Bagai R K and Vikram Kumar 1994 Bull. Mater. Sci. 171057

Venkataraghavan R, Rao K S R K, Sreedhar A K and Bhat H L 1996 J. Appl. Phys. (Communicated) van Welzenis R G, van Setten F M and Schannen OF Z 1991 Appl. Phy's. A52 19

Williams G M, Whitehouse C R, Chew N G, Blackmore G W and Cullis A G $1985 \mathrm{~J}$. Vac'. Sci. Tec'hnol. B3 704

Zahn D R T, Richter W. Eickhoff T, Geurts J, Golding T D. Dinan J H. Mackey K J and Williams R H 1989 Appl. Surf. Sci. $\mathbf{4 1 / 4 2 ~} 497$ 